tal Reserve for our Government and we predict the establishment of the most efficient service in this important department of any nation.

Caring for the mouths of applicants for enlistment who are unable to pay for dental service is a most important object of the League and we wish to take this opportunity to thank our members for the unparalleled response this phase of our worlk has received. We are proud of our profession and when the final reckoning shall come, it will be shown that we have saved our country many thousands of fighters. The League has been designated the official medium for prosecuting this work and members are requested to report to our headquarters all cases thus cared for so that we may compile statistics to present to the Surgeon General. The Government appreciates what we are doing and we believe our efforts will not go unrewarded when future favorable legislation may be desired by us.

We hope to bring many new members into the National Dental Association thru the League and would call the attention of the Officirs of our Units to this matter. We should bring many into the National before the annual meeting in october next.

The second annual meeting of the League will be held in conjunction with the National Dental Association on October 23rd, 1917, in Concert Hall, Hotel Astor, New York. We are planning a splendid program and will be able to report wonderful results from the different Units. Several dental ambulances for use in France are being supplied by our Units and other equally patriotic movements are under way.

We urge the formation of Units more generally in order to promote the various objects of the League. Organization is essential and we would point out that now is the time our best efforts are demanded as the League is organized as a war measure, therefore, we invite correspondence with this object in view. Headquarters are at 131 Allen st., Buffalo, N. Y.

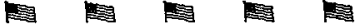

\section{OUR DENTAL MOTOR AMBULANCES.}

\author{
By J. W. Beach, Chairman, Buffalo, N. Y.
}

The League is happy to announce to iis members the remarkable success of its plans for supplying dental ambulances for service in France. The Surgeon General already has accepted three ambulances presented by the San Francisco and other Units of Northern California. The Southern California district of the League will soon follow with a like number. These are the first ambulances to be tendered the Government.

One of the most striking incidents of this movement recently occurred in New York City when Dr. Charles F. Ash received a magnificent donation from an appreciative patient of $\$ 10,000$ for five dental ambulances for the League. This is a splendid testimonial of the esteem in which Dr. Ash is held by his patients and an equally alued recognition of the great benefit to suffering humanity which the dental ambulance supplies.

We will gladly give to the Cleveland Unit the credit of originating the dental ambulance idea in connection with the League. At the first meeting of the Committee on Dentistry on April 15th, an offer of an ambulance was made to the Government by the Cleveland and other Ohio Units. Funds have been raised and the construction is now under way.

Kansas City and several other Units 
about the country are following the commendable example of cleveland. Should the League find the demand sufficient, at least twenty-five ambulances could be secured.

Two styles of dental ambulances may be recommended. The first embodies a full equipment permanently mounted on a Ford chassis enclosed in a roomy body with sufficient light. The other style is portable and may be set up under a tent. Both kinds have about an equal number of advantages and disadvantages, therefore, we recommend the supply to be in like proportion.

The value of the dental ambulance in war is almost beyond computation, for it can be driven close to the trenches and render a service of untold value. Dr. John D. Millikin, of San Francisco, says: I suggested that four dentists and two mechanics, these latter to act as chauffeurs, with one ambulance, to make up one Dental Motor Ambulance Unit. Two dentists and one mechanic could work at
Base Hospitals while two dentists and one mechanic visited the rest stations with the ambulance, thus furnishing relief for the poor fellows coming back to the rest stations from the trenches."

The League will gladly furnish specifications to those interested and will assist in every way possible to forward this splendid plan. No more valuable service can be rendered by our profession and we hope to see a well trained Dental Motor Ambulance Unit accompany each one to France. This will insure the fullest degree of usefulness and more clearly emphasize the magnanimous spirit of helpfulness which is actuating the whole dental profession today.

At the annual meeting of the League next October, both styles of ambulance will be shown, as well as everything of interest connected with our service with the Officers Reserve Corps. It is to be a "Preparedness Exhibit" and we believe it will prove one of the most interesting features of the meeting of the National Dental Association.

\title{
AN IMPERATIVE COMMISSION FOR EVERY DENTIST.
}

\author{
By J. W. Beach, Chairman, Buffalo, N. Y.
}

The Preparedness League of American Dentists is directed by Dr. W. H. G. Logan, Chairman of the Committee on Dentistry, General Medical Board, Council of National Defense, to present to the Committee at its first meeting in September, as complete a list as can be obtained of recruits and prospective recruits made dentally fit during August.

Such a list will impress our Government with the great importance of our special service and give tangible evidence of the splendid spirit of cooperation and of sacrifice so strikingly shown by our profession during the recent months.
Every dentist has just cause for pride in the fact that fully 25,000 applicants for enlistment in the Army, Navy and members of the National Guard have been dentally equipped to fight for our country, thru the gratuitous service of thousands of members of our great profession.

\section{WE WILL DO OUR PART.}

The Present Emergency demands our best efforts! Every man sworn to fight for our colors should have his mouth and teeth put in proper state for three years service under the conditions now existing in France. This demand for our service 